\title{
A Low Voltage Wide-Input-Range Bulk-Input CMOS OTA
}

\author{
IVAN GRECH, JOSEPH MICALLEF, GEORGE AZZOPARDI AND CARL J. DEBONO \\ Department of Microelectronics, University of Malta, Msida MSD 06, Malta Tel.: +356 2340 2511, Fax: +356 21343577 \\ E-mail:igrech@eng.um.edu.mt
}

Received May 31, 2004; Accepted October 15, 2004

\begin{abstract}
The $G_{m}-C$ technique is extensively used for continuous-time filtering applications because it results in tunable, wideband and compact designs. In this paper, an OTA architecture using a novel bulk-input differential pair without the use of a tail current source is proposed. Good CMRR is still achieved by using the gate terminal to control the total current in the differential pair, via the use of a dummy pair. The OTA also exhibits a wide differential input range and good $G_{m}$-tunability. For this design, two standard double-poly double-metal CMOS processes were investigated: a $0.8 \mu \mathrm{m}$ process having a nominal threshold voltage of around $0.7 \mathrm{~V}$ and a $0.35 \mu \mathrm{m}$ process having a nominal threshold voltage of $0.5 \mathrm{~V}$. Simulation results are presented for both designs while test results are presented, for the OTA, implemented using the $0.8 \mu \mathrm{m}$ process, used in a second order cochlea low-pass filter.
\end{abstract}

Key Words: low voltage, bulk-input, OTA, CMOS design

\section{Introduction}

OTAs are a fundamental building block in analog signal processing applications and in particular in $G_{m}-C$ filters. Low voltage operation is an important issue due to low power consumption requirements in battery operated applications and also for compatibility with today's low supply voltages used for digital applications [1]. A low voltage OTA is thus proposed here, with particular application to $G_{m}-C$ filters, although other applications could include op amps and automatic gain control circuits. The concept of using the bulk terminal of an MOS device as input in a differential pair has already been explored [2-4]; however, these schemes still utilize a tail current source. A further reduction in the supply voltage requirement is achieved here via the elimination of the tail current source, thus reducing the supply voltage requirement by around $0.2 \mathrm{~V}$, without compromising the common mode rejection ratio (CMRR).

\section{Implementation}

Figure 1(a) shows a conventional differential input stage, where the common mode input range is ( $V_{\mathrm{DSsat} 4}-$ $\left.\left|V_{\mathrm{TP}}\right|\right)$ to $\left(V_{\mathrm{DD}}-\left|V_{\mathrm{DSsat} 3}\right|-\left|V_{\mathrm{GS} 1}\right|\right)$, where $V_{\mathrm{DSsat} 4}$ is the saturation voltage for $I_{\mathrm{B} 2}$. Taking a supply voltage of $0.9 \mathrm{~V}, V_{T}=0.7 \mathrm{~V}$ and $V_{\text {DSsat }}=0.1 \mathrm{~V}$, the input range would be -0.6 to $0 \mathrm{~V}$. This means that the OTA is not suitable for $G_{m}-C$ filters, since it is not possible to provide the required voltage shift between one stage and another.

The circuit shown in Fig. 1(b) provides a solution to this problem since the input is applied to the bulk and therefore the common mode input range is only limited by the leakage current through the drain-bulk and source-bulk diodes, which effectively amounts to a common mode input range of 0.3 to $0.9 \mathrm{~V}$. The minimum supply voltage for this input stage is $V_{\mathrm{DSsat} 5}+V_{\mathrm{GS} 1}$, where $V_{\mathrm{DSsat} 5}$ is the saturation voltage of $I_{\mathrm{B} 1}$, thus allowing operation down to a supply voltage of 0.9 V. In Fig. 1(b), $M_{1}-M_{4}$ are matched. $M_{1}-M_{2}$ form a dummy pair which mirrors the operation of $\mathrm{M}_{3}-\mathrm{M}_{4}$. In this configuration, $I_{1}+I_{2}$ is kept constant and equal to $I_{\mathrm{B} 1}$, by controlling the gate voltage: in this way, the current $I_{3}+I_{4}$ is also kept equal to $I_{\mathrm{B} 1}$, which ensures an adequate CMRR. Considering a single-ended output, with a differential input signal, the voltage gain of the circuit shown in Fig. 1(b) is given by:

$$
A_{v d}=\frac{g_{m b}}{2\left(g_{d s 3}+g_{b 2}+g_{L}\right)}
$$




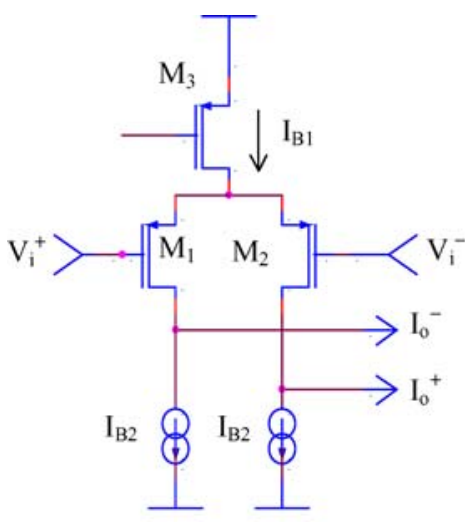

(a)

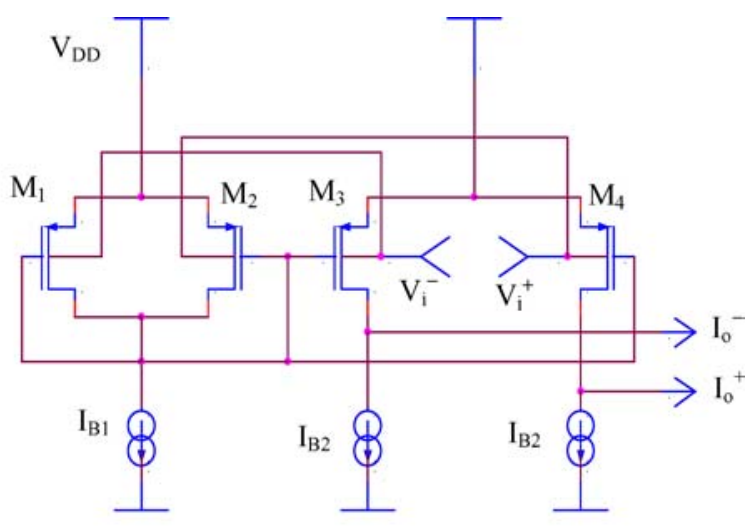

(b)

Fig. 1. (a) Conventional and (b) low voltage bulk-driven differential input stage.

where $g_{b 2}, g_{L}$ are the output conductance of the current source $I_{B 2}$ and load, respectively. For a common mode input signal, the corresponding single-ended output voltage gain is given by:

$$
A_{v c}=\frac{g_{m b}\left(g_{d s 1}+g_{b 1} / 2\right)}{g_{m}\left(g_{L}+g_{d s 3}\right)}
$$

where $g_{b 1}$ is the output conductance of current source $I_{B 1}$. The single-ended output CMRR of the differential input stage is therefore given by:

$\mathrm{CMRR}=\frac{A_{v d}}{A_{v c}}=\frac{g_{m}\left(g_{L}+g_{d s 3}\right)}{2\left(g_{d s 3}+g_{b 2}+g_{L}\right)\left(g_{d s 1}+g_{b 1} / 2\right)}$

Thus a high CMRR value, comparable to that of a conventional differential pair can be obtained provided $g_{d s 1}$ and $g_{b 1}$ are kept sufficiently small. In order to achieve a high CMRR, it is important that the dummy differential pair $M_{1}, M_{2}$ and the functional differential pair $M_{3}, M_{4}$ are accurately matched: common centroid layout techniques have to be used for this purpose.

Figure 2 shows the complete OTA with the necessary current mirrors in order to transform the differential outputs into a single-ended output. A cascode output stage is used in order to enhance the output resistance of the OTA. In this OTA, $(W / L)_{11}=$ $(W / L)_{13},(W / L)_{12}=(W / L)_{14},(W / L)_{9}=(W / L)_{10}$. Thus the effective transconductance of the OTA is given by:

$$
G_{m}=g_{m b 3,4} \cdot \frac{(W / L)_{12}}{(W / L)_{11}}
$$

\section{Simulation Results}

\subsection{OTA Simulation Results for the $0.8 \mu \mathrm{m}$ CMOS Process Implementation}

Figure 3 shows the DC transfer characteristic of the OTA of Fig. 1(b) for differential bias currents $I_{B 1}$ ranging from $100 \mathrm{nA}$ to $1 \mu \mathrm{A}$. The common mode input voltage was kept at $450 \mathrm{mV}$, resulting in an input linear range of around $\pm 600 \mathrm{mV}$ which is significantly higher than the value of $60 \mathrm{mV}$ that is typically achievable using a standard differential pair [5]. This is due to the fact that the bulk transconductance is lower than the gate transconductance. Figure 4 shows the corresponding $G_{m}$-value versus frequency plot. Since the differential pair operates in weak inversion, it can be seen that $G_{m}$ is proportional to the bias current, although at high bias currents some compression is noticed due to the transistors starting to operate in moderate inversion.

Figure 5 shows the input referred noise plot with frequency for an $I_{B}$ range from 0.1 to $1 \mu \mathrm{A}$. The corresponding thermal noise density range is $2.2 \mu \mathrm{V} / \sqrt{ } \mathrm{Hz}$ to $820 \mathrm{nV} / \sqrt{ } \mathrm{Hz}$. The higher noise value is expected at low bias currents. When compared to gate-driven MOS transistors, bulk-driven MOS transistors exhibit the same channel noise current; however, the input referred noise for bulk-driven MOS transistors is higher 


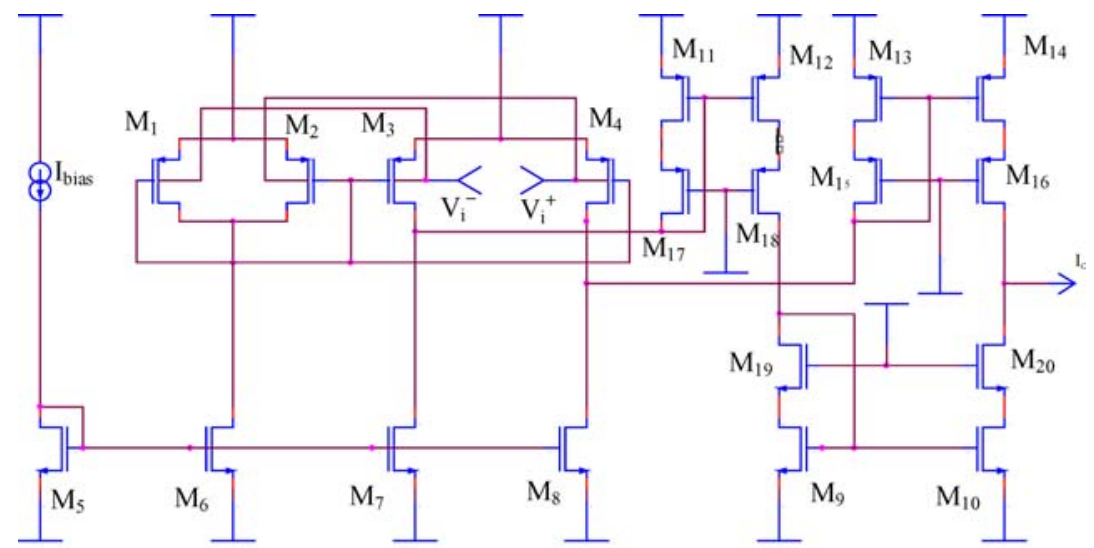

Transistor sizes: $\mathrm{M}_{1-4}, \mathrm{M}_{11-18}: \mathrm{W}=80 \mu \mathrm{m} ; \mathrm{L}=2 \mu \mathrm{m}$.

$\mathrm{M}_{5-10}, \mathrm{M}_{19-20}: \mathrm{W}=50 \mu \mathrm{m} ; \mathrm{L}=2 \mu \mathrm{m}$.

Fig. 2. Complete bulk-input OTA.

DC Response

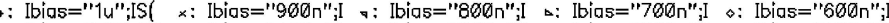

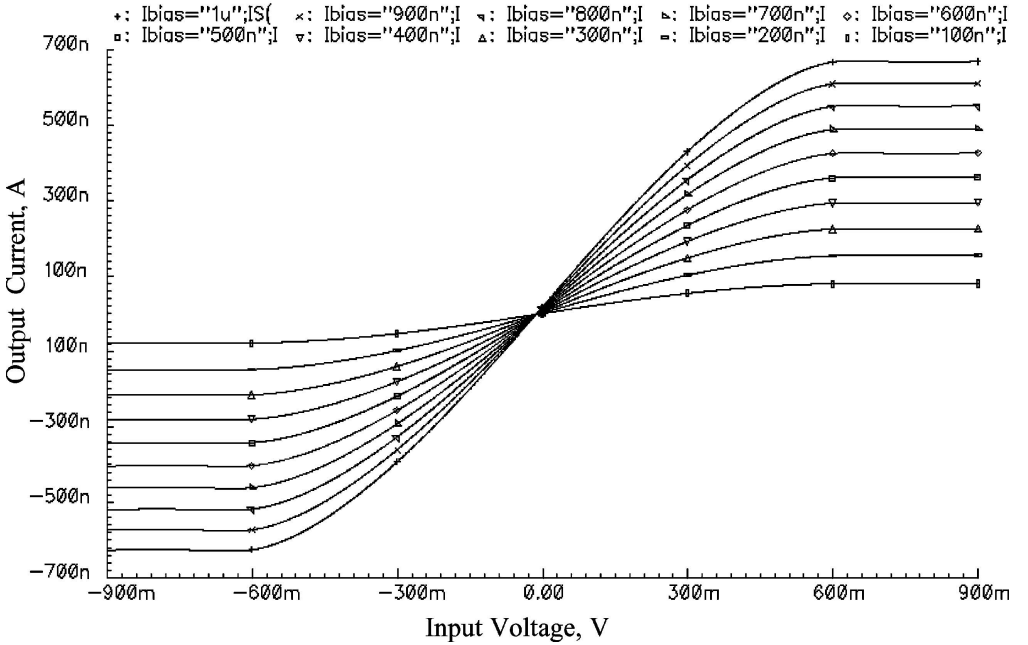

Fig. 3. DC Transfer characteristics of the OTA for $I_{B}=0.1$ to $1 \mu \mathrm{A}$.

since the bulk transconductance is lower than the gate transconductance.

\subsection{Second Order LPF Simulation Results}

The second order cochlea LPF [5] shown in Fig. 6, using the proposed OTA was simulated. For this section, the transfer function is given by:

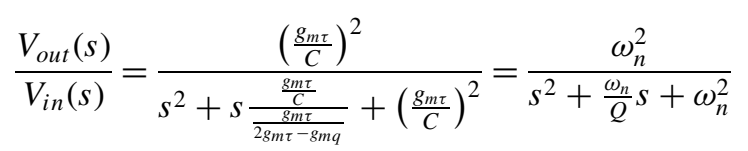

where $g_{m \tau}$ is the transconductance of OTA 1, OTA $_{2}$ and $g_{m q}$ is the transconductance of $\mathrm{OTA}_{3}$. In the standard second order LPF expression, $\omega_{n}=g_{m \tau} / C$ and $Q=$ $g_{m \tau} /\left(2 g_{m \tau}-g_{m q}\right)$. Since the input transistors operate in weak inversion, their transconductance is proportional to the bias current.

Figure 7 shows the frequency response of the second order filter for the $\omega$-tuning current $\left(I_{\omega}\right)$ in the range $5 \mathrm{nA}$ to $1 \mu \mathrm{A}$. Using $C=10 \mathrm{pF}$, the peaking frequency ranges from $186 \mathrm{~Hz}$ to $23 \mathrm{kHz}$. The $Q$-tuning current $I_{Q}$ was kept equal to $1.5 I_{\omega}$ in all cases, resulting in a theoretical $Q$-factor of 2 . In practice, the $Q$-factor obtained is 1.6: this value is lower than the theoretical value due 


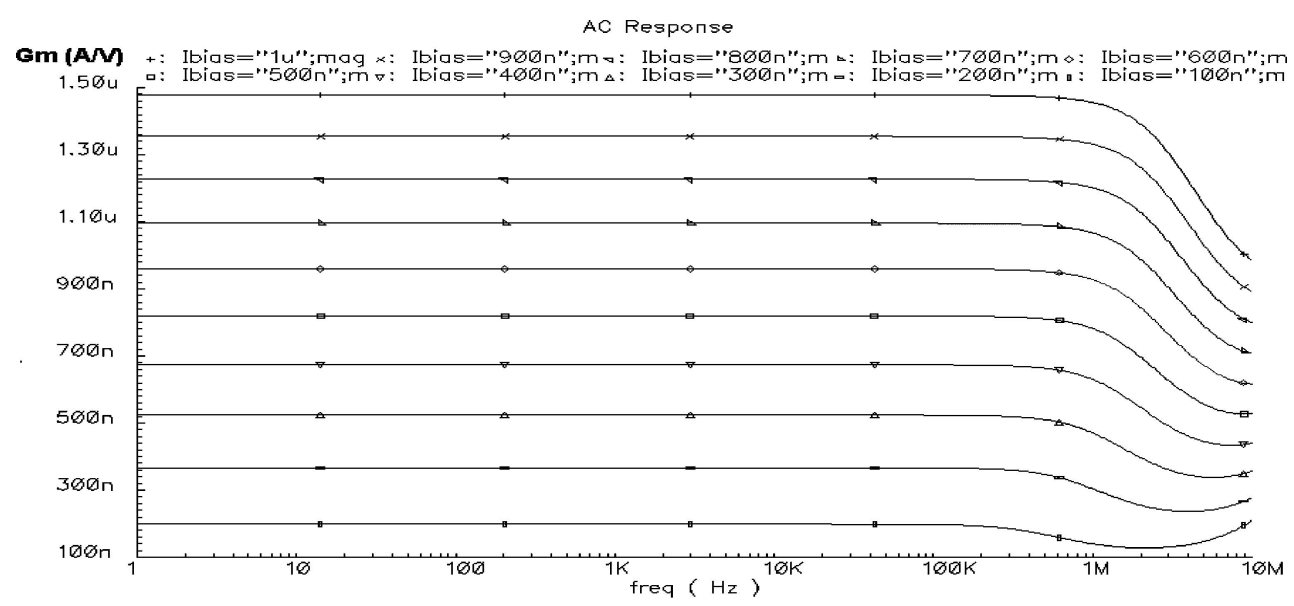

Fig. 4. AC response of the OTA for $I_{B}=0.1$ to $1 \mu \mathrm{A}$, measured using a load resistor of $1 \Omega$.

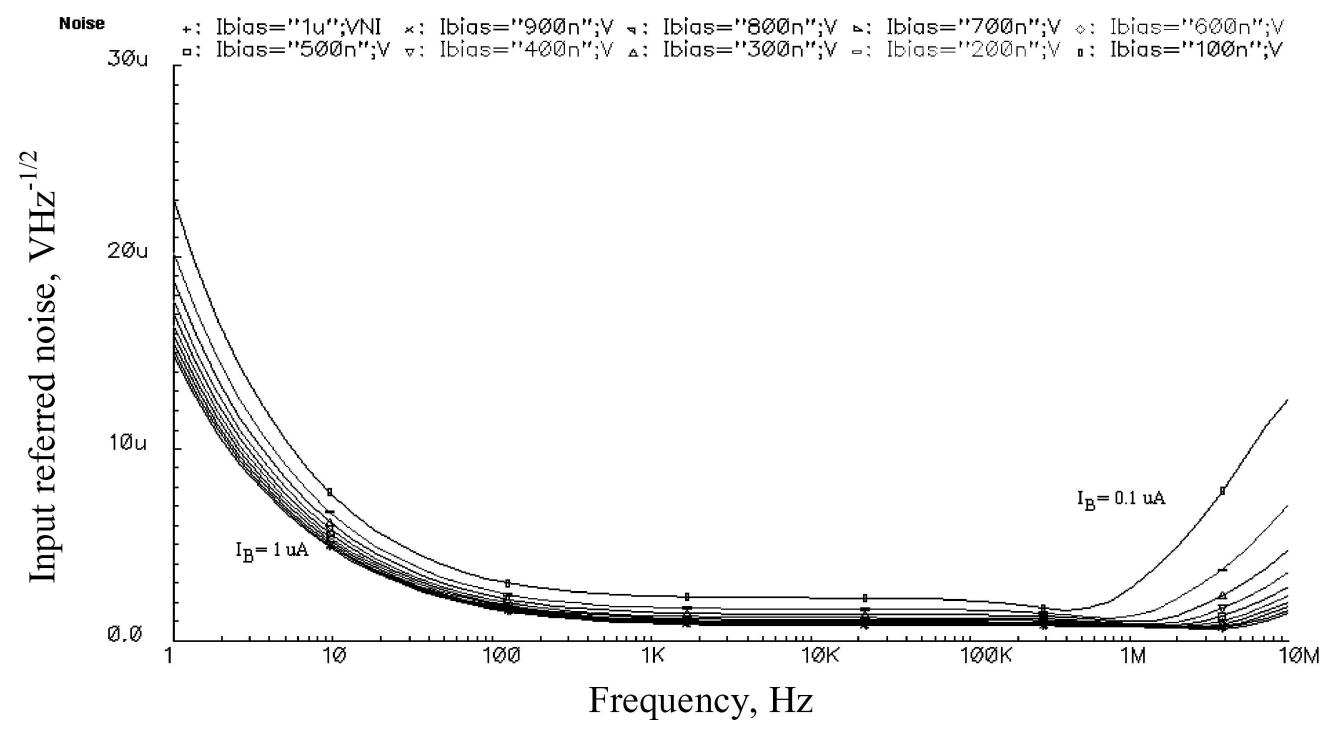

Fig. 5. Noise performance of the open circuit OTA for $I_{B}=0.1$ to $1 \mu \mathrm{A}$.

to the finite input resistance of the driven bulk-input OTA and also due to the finite output resistance of the driver OTA itself.

\subsection{OTA Simulation Results for the $0.35 \mu \mathrm{m}$ CMOS Process Implementation}

In order to investigate the possibility of further lowering the supply voltage, the same OTA architecture was also characterized using a $0.35 \mu \mathrm{m}$ CMOS process having a threshold voltage of around $0.5 \mathrm{~V}$ : the transistor gate widths were kept the same as for the $0.8 \mu \mathrm{m}$ process, but the gate lengths were decreased to $1 \mu \mathrm{m}$. The DC transfer characteristics of the OTA, implemented in the $0.35 \mu \mathrm{m}$ process and operated at a supply voltage of $0.7 \mathrm{~V}$, for the differential pair bias current $I_{B 1}$ ranging from $100 \mathrm{nA}$ to $1 \mu \mathrm{A}$, are shown in Fig. 8. The common mode input voltage is kept at $350 \mathrm{mV}$, resulting in an input linear range of around $\pm 350 \mathrm{mV}$; this value is lower than that obtained for the $0.8 \mu \mathrm{m}$ implementation, mainly because of the higher $W / L$ ratio used in this case and the higher intrinsic transconductance $(K)$ of the $0.35 \mu \mathrm{m}$ process. Figure 9 shows the corresponding $G_{m}$-value versus frequency plot; this plot is very 


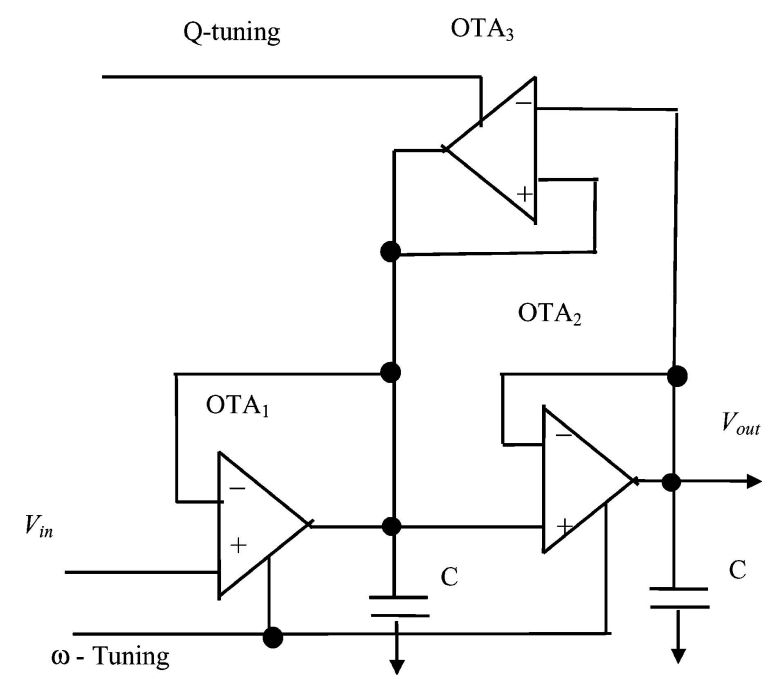

Fig. 6. Second order LPF with separate cut-off frequency and $Q$ factor tuning.

similar to that obtained for the $0.8 \mu \mathrm{m}$ process; however, a higher $G_{m}$-value can be observed again because of the higher values of $W / L$ and $K$ parameter.

Table 1 summarizes the performance of the OTA implemented in the $0.35 \mu \mathrm{m}$ process, at different supply voltages with a load capacitance of $0.5 \mathrm{pF}, I_{\text {bias }}=1 \mu \mathrm{A}$ and an input common mode voltage set at $V_{\mathrm{DD}} / 2$. A high CMRR is achieved at $0.7 \mathrm{~V}$ and $0.8 \mathrm{~V}$ even though a tail current source is not used. At $V_{\mathrm{DD}} \geq 0.9 \mathrm{~V}$, bulk leakage in the input transistors becomes more appreciable and this results in some degradation of the OTA parameters mainly the CMRR, together with an increase in the input offset voltage. Figure 10 shows the variation of (a) the AC response and (b) the common mode gain, of the OTA, with different common mode input voltages with the OTA operated at $I_{\text {bias }}=1 \mu \mathrm{A}$ and $V_{\mathrm{dd}}=0.7 \mathrm{~V}$. Results show that for $V_{\mathrm{DD}}=0.7 \mathrm{~V}$ the OTA exhibits very little parameter dependence on the input common mode voltage as regards to its AC performance; however, a $15 \mathrm{~dB}$ degradation in the CMRR can be noticed as the common mode voltage is increased from 0 to $700 \mathrm{mV}$.

\subsection{Third Order Butterworth LPF Simulation Results}

A third order Butterworth filter, based on the second order cochlea LPF, discussed in Section 3.2, followed by a first order section, and using the OTA implemented in $0.35 \mu \mathrm{m}$ technology, has been simulated. Figure 11(a) shows the frequency response of the third order filter for

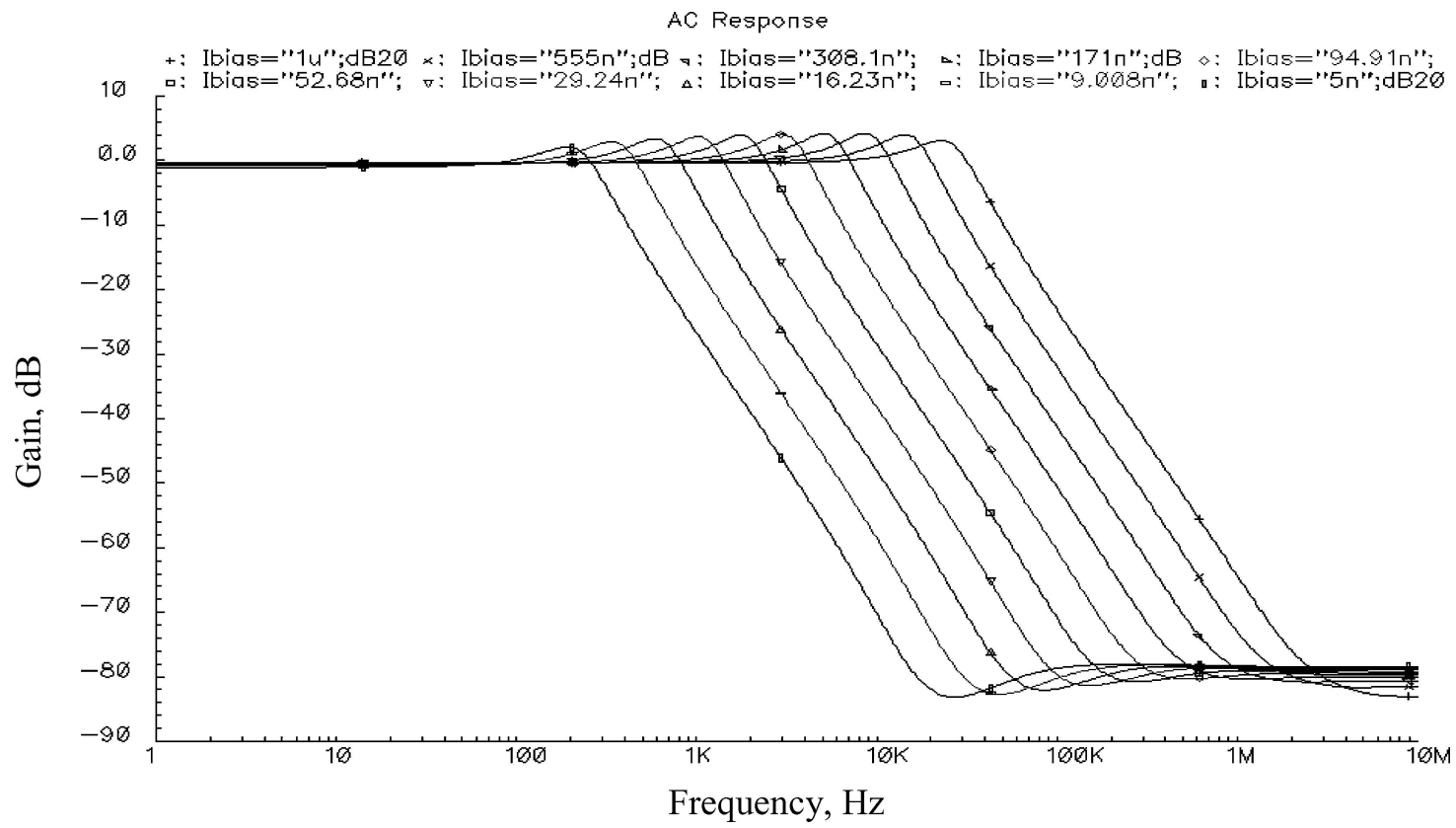

Fig. 7. Frequency response of the 2 nd order LPF for $I_{\omega}=5 \mathrm{nA}$ (left curve) to $1 \mu \mathrm{A}$ (right curve) with $I_{Q}=1.5 I_{\omega}$. 
Table 1. Simulation results for the $0.35 \mu \mathrm{m}$ process OTA.

\begin{tabular}{lcccccc}
\hline Supply voltage $(\mathrm{V})$ & GBW $(\mathrm{kHz})$ & $\mathrm{DC}$ gain $(\mathrm{dB})$ & Phase margin $\left({ }^{\circ}\right)$ & Slew rate $(\mathrm{V} / \mu \mathrm{s})$ & Power $(\mu \mathrm{W})$ & $\mathrm{CMRR}(\mathrm{dB})$ \\
\hline 0.7 & 446 & 63 & 67 & 1 & 3.5 & 123 \\
0.8 & 450 & 70 & 65 & 1.1 & 4 & 131 \\
0.9 & 398 & 69 & 66 & 0.84 & 4.5 & 91 \\
1.0 & 371 & 63 & 68 & 0.83 & 5 & 66 \\
\hline
\end{tabular}

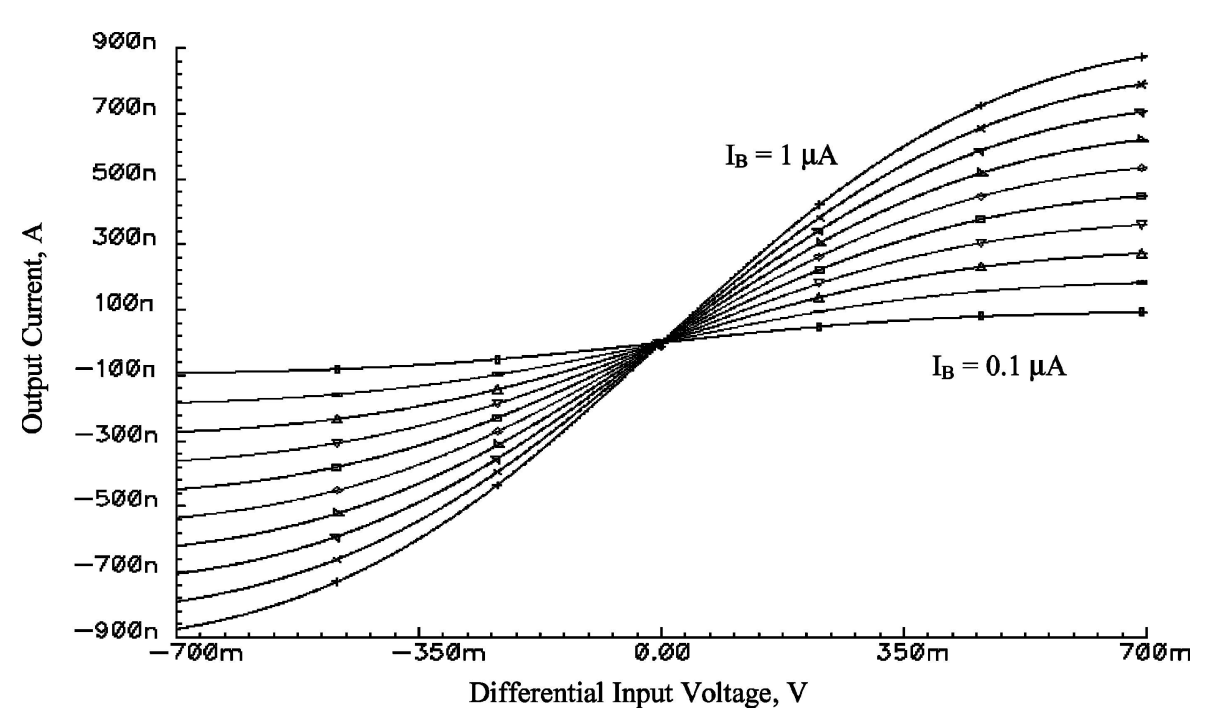

Fig. 8. DC Transfer characteristics of the OTA operated at $\mathrm{V}_{\mathrm{DD}}=0.7 \mathrm{~V}$ for $I_{B}=0.1$ to $1 \mu \mathrm{A}$ in steps of $100 \mu \mathrm{A}$.

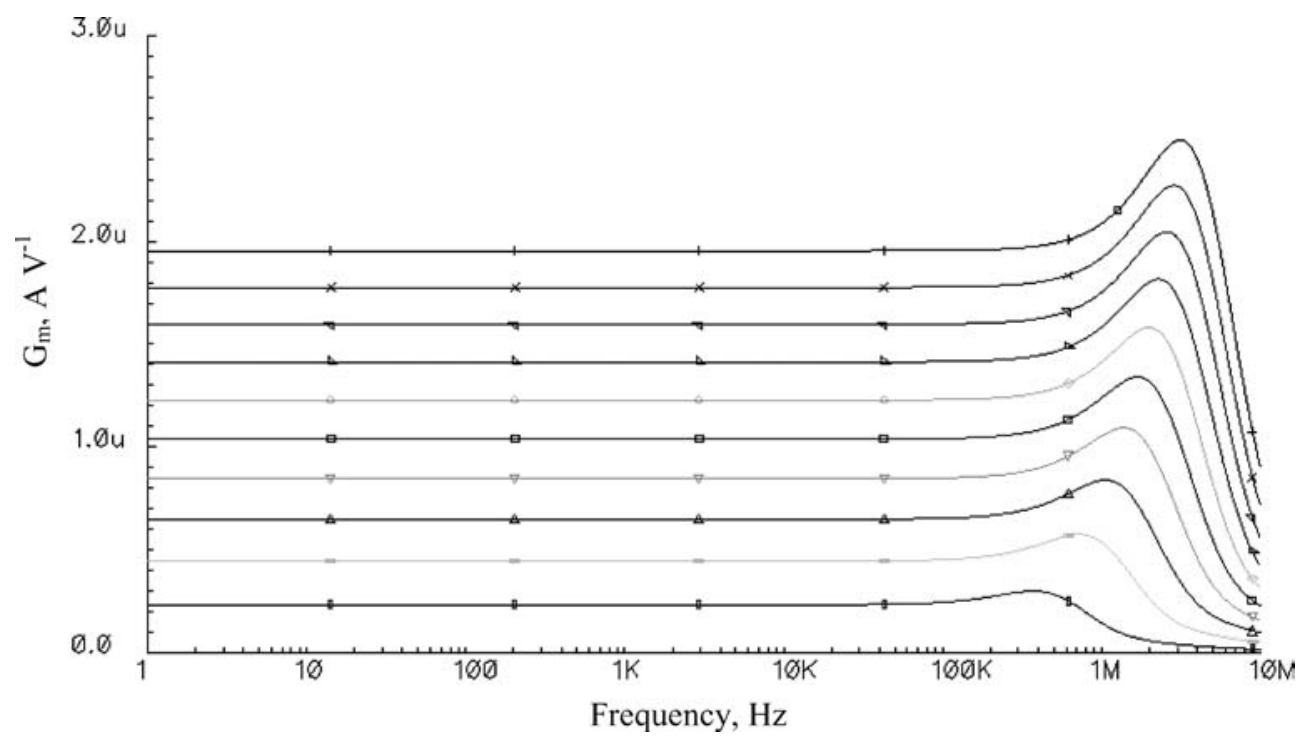

Fig. 9. AC response of the OTA at $V_{\mathrm{DD}}=0.7 \mathrm{~V}$ for $I_{B}=0.1 \mu \mathrm{A}$ (lower curve) to $1 \mu \mathrm{A}$ (upper curve) in steps of $100 \mathrm{nA}$, measured using a load resistor of $1 \Omega$. 

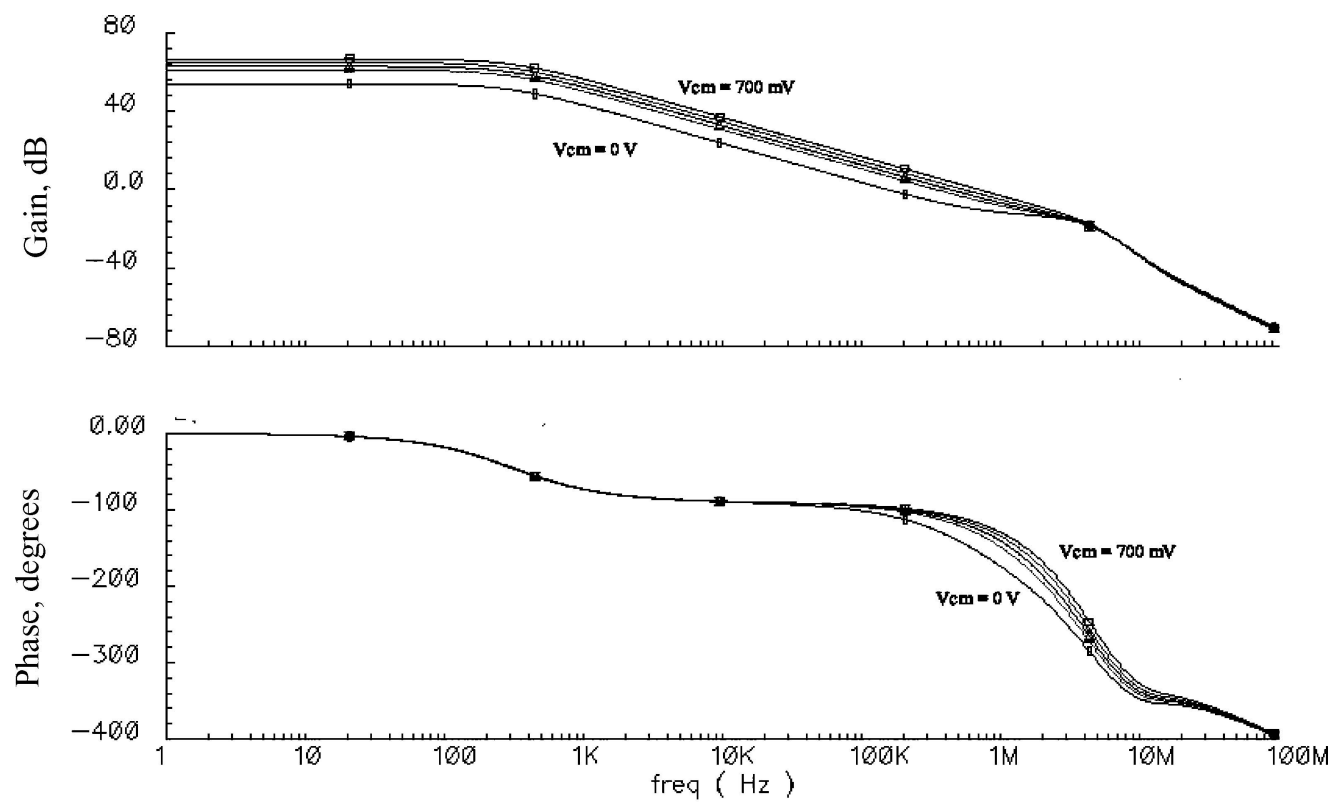

(a)

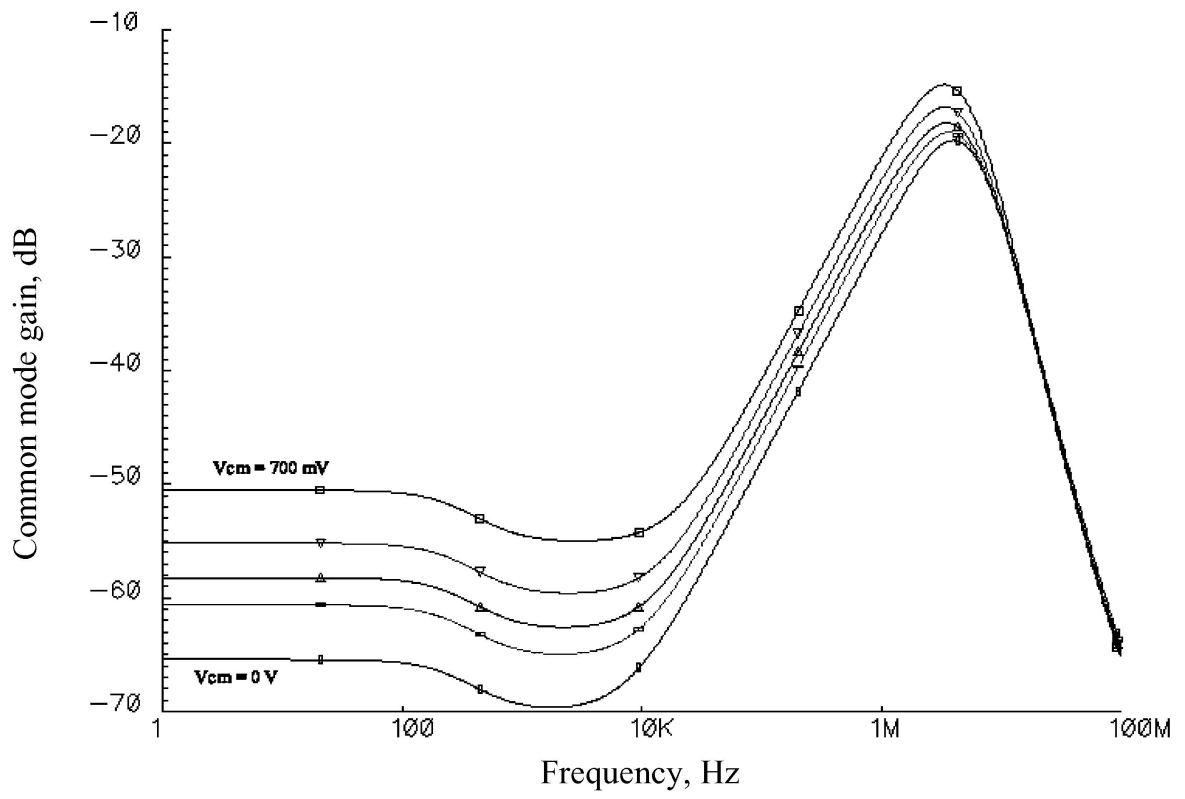

(b)

Fig. 10. Variation of the (a) differential gain and phase response and (b) common mode gain with common mode input voltage ranging from 0 to $700 \mathrm{mV}$. 


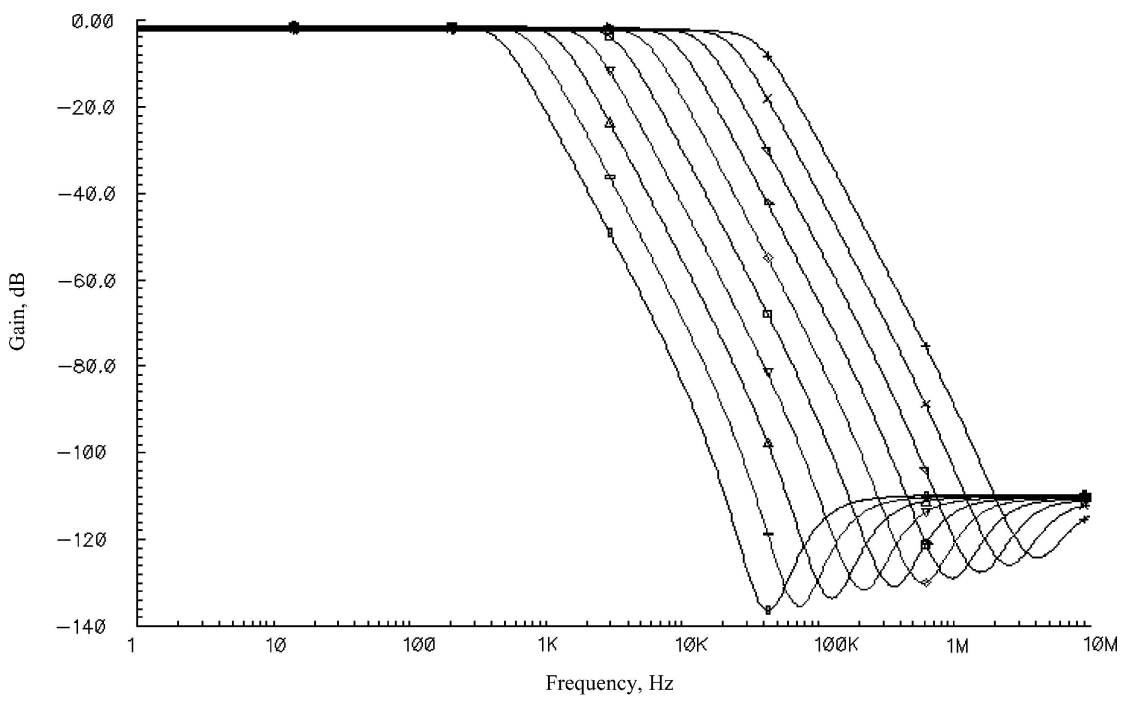

(a)

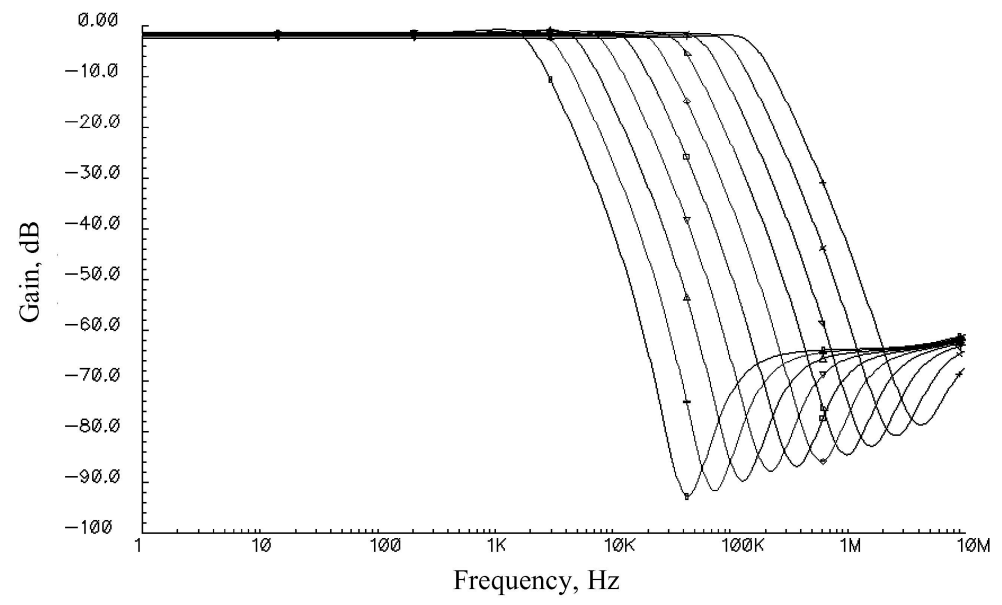

(b)

Fig. 11. Butterworth LPF implemented using a second order stage followed by a first order stage, for tuning currents swept logarithmically from $10 \mathrm{nA}$ (left curve) to $1 \mu \mathrm{A}$ (right curve) for (a) filter capacitors set to $10 \mathrm{pF}$ and (b) parasitic capacitors only.

the $\omega$-tuning current in the range $10 \mathrm{nA}$ to $1 \mu \mathrm{A}$. With the filter capacitors set to $10 \mathrm{pF}$, the cut-off frequency ranges from $500 \mathrm{~Hz}$ to $38 \mathrm{kHz}$, with a stop band attenuation of $-110 \mathrm{~dB}$. It is evident that the cut-off frequency is linearly related to the tuning current- - this is expected since the cut-off frequency is proportional to the $G_{m}$ of the OTA, which in turn is proportional to the tuning current if the MOS transistors operate in weak inversion. The same simulation carried out without using any capacitors, but relying on the bulk input parasitic and the corresponding results, are shown in Fig. 11(b). In this case, the cut-off frequency ranges from 1.82 to
$200 \mathrm{kHz}$ for the same bias current range; however, the stop band attenuation is limited to $-62 \mathrm{~dB}$, since the filtering capacitors are of the same order of magnitude as the other parasitic capacitors in the circuit.

\section{Measurement Results for the $0.8 \mu \mathrm{m}$ Implementation}

The OTA designed using the $0.8 \mu \mathrm{m}$ process was fabricated and tested. The microphotograph of the test chip consisting of a single OTA is shown in Fig. 12. Three 


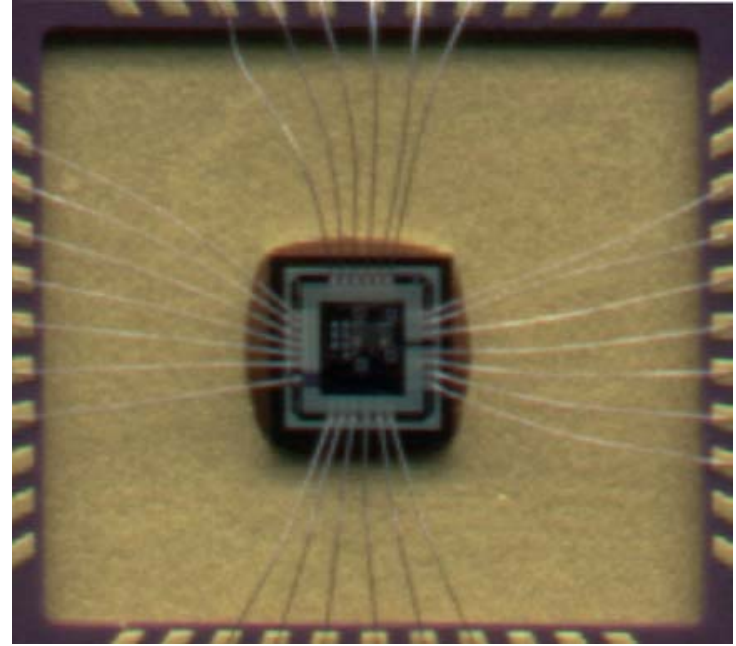

Fig. 12. Microphotograph of the OTA test chip. test chips were then wired as a cochlea second order LPF, with two external $10 \mathrm{pF}$ capacitors, and the corresponding measured frequency responses for $I_{B}=5 \mathrm{nA}$ to $1 \mu \mathrm{A}$ are shown in Fig. 13 for (a) feedback OTA disabled, (b) feedback OTA current enabled with $I_{Q}=1.5$ $I_{\omega}$. For the case when the feedback OTA is disabled, the $Q$-factor is equal to 0.5 , resulting in a critically damped second order filter: in this case the measured results are very close to the theoretical response. For the second case, the measured $Q$-factor of 1.4 is lower than the theoretical value and is probably influenced by the leakage current to the bulk inputs of the OTAs.

\section{Conclusion}

Simulation and test results, for both the 0.8 and $0.35 \mu \mathrm{m}$ CMOS processes, indicate that the differential pair

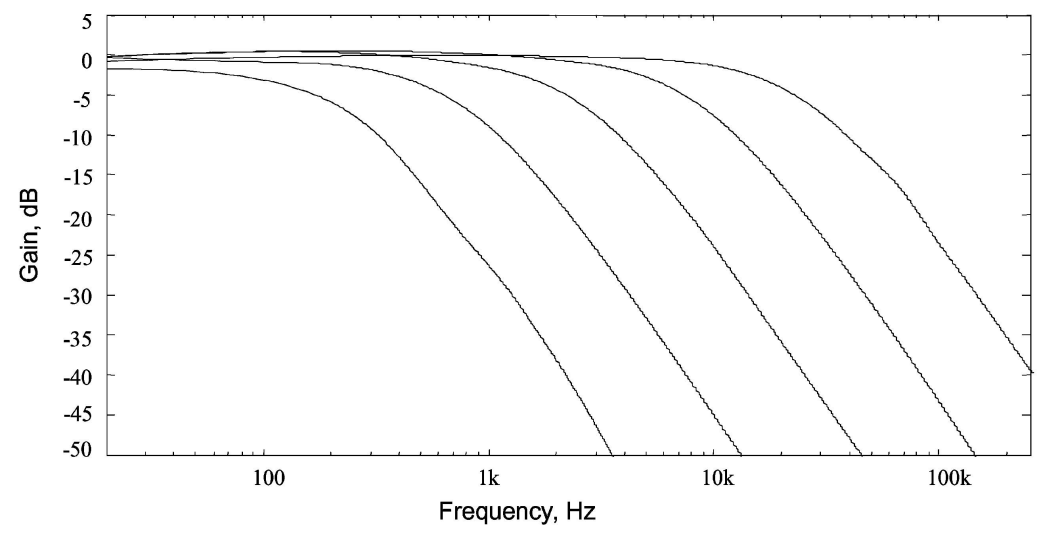

(a)

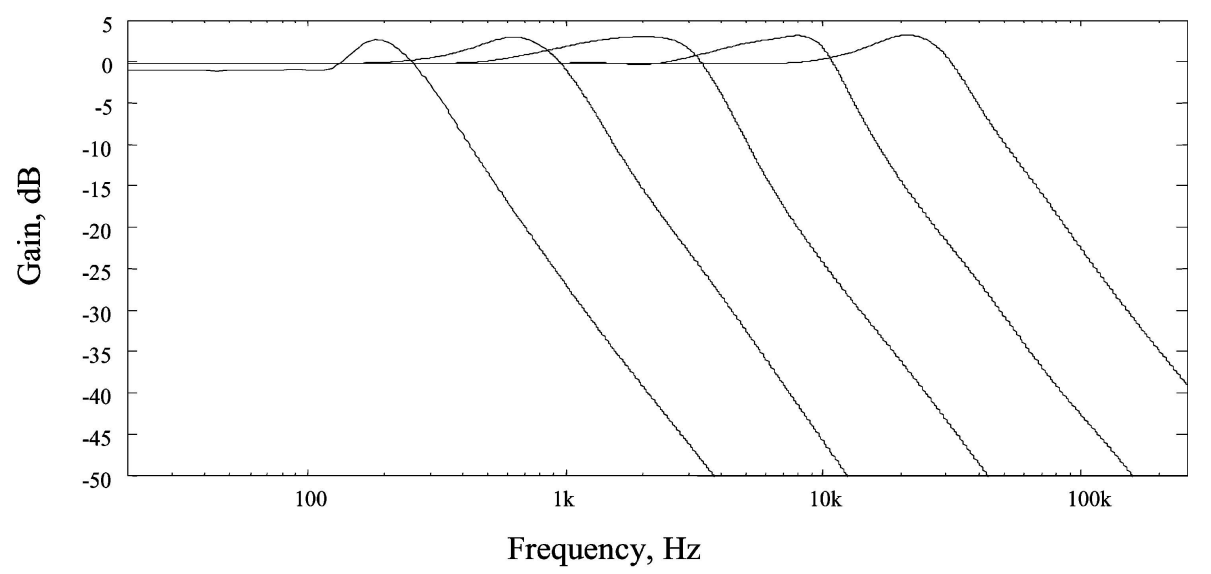

(b)

Fig. 13. Frequency response (starting from left curve) for $I_{B}=5,18,71,260,1000 \mathrm{nA}$, for (a) feedback OTA disabled and (b) feedback OTA enabled with $I_{Q}=1.5 I_{\omega}$. 
using the bulk terminal as input and the gate terminal for CMRR control is a feasible solution in the area of very low voltage applications-operation supply voltages down to $0.7 \mathrm{~V}$ is achievable using a process having a threshold voltage of $0.5 \mathrm{~V}$. When used as the input stage of an OTA, the differential pair exhibits a wide linear input range due to its intrinsically low transconductance value, and is thus applicable to a wide range of current mode analog signal processing techniques, where a slight input bias current can be tolerated as for example in most filtering applications. A second order $G_{m}-C$ filter has been implemented using three bulk-input OTAs and test chip results presented.

\section{References}

1. S. Yan and E. Sanchez-Sinencio, "Low voltage analog circuit design techniques: A tutorial." IEICE Trans. Fundamentals, vol. E83-A, no. 2, pp. 179-196, 2000.

2. R. Sarpeshkar, R.F. Lyon, and C. Mead, "A low-power widelinear-range transconductance amplifier." Analog Integrated Circuits and Signal Processing, vol. 13, pp. 123-151, 1997.

3. K. Lasanen, E. Raisanen-Ruotsalainen, and J. Kostamovaara, "A 1-V $5 \mu \mathrm{W}$ CMOS-Opamp with bulk-driven input transistors," in Proc. $43^{\text {rd }}$ IEEE Midwest Symp. on Circuits and Systems, Lansing MI, Aug. 8-11, pp. 1038-1041, 2000.

4. B.J. Blalock, P.E. Allen, and G.A. Rincon-Mora, "Design of 1-V op amps using standard digital CMOS technology." IEEE Trans. Circuits \& Syst. II: Analog and Digital Signal Processing, vol. 45, no. 7, pp. 769-780, 1998.

5. L. Watts, D.A. Kerns, and R.F. Lyon, "Improved implementation of the silicon cochlea." IEEE Journal on Solid-State Circuits, vol. 27, pp. 692-700, 1992.

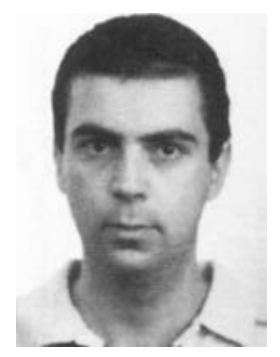

Ivan Grech received his B.Eng.(Hons.) degree in 1993 and M.Sc. in 1996 from the University of Malta.
In 1994 he joined the Department of Microelectronics at the University of Malta where he is employed as a lecturer. He received the Ph.D. degree from the University of Surrey, U.K. in 2002. His research interest is in CMOS analog integrated circuit design.

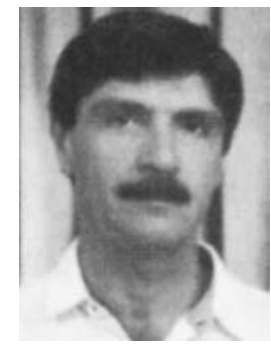

Joseph Micallef received his B.Sc. Eng(Hons.) degree in electronics engineering from the University of Malta in 1972, and M.Sc. and Ph.D. degrees in electrical engineering from the University of Surrey, U.K., in 1989 and 1993, respectively. From 1973 to 1981, he was with General Instruments, engaged in work on high voltage components and circuits and on IFTs. He moved to SGS-THOMSON Microelectronics in 1981 where he was involved with packaging of MOS ICs. In 1989, he joined the Faculty of Engineering at the University of Malta and is now lecturer in the Department of Microelectronics. His current research activities include analog integrated circuit design, as well as optical properties of III-V quantum well structures.

George Azzopardi photo/biography not available upon publication.

Carl J. Debono photo/biography not available upon publication. 